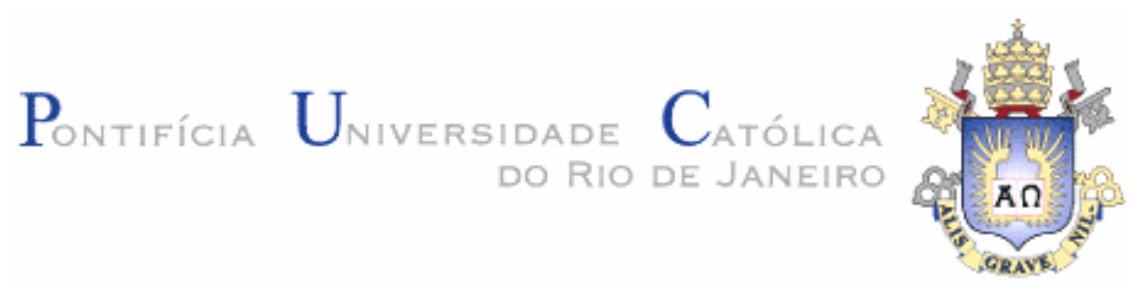

Carmen Julia Ayquipa Huaman

Simulação 3D pelo Método dos Elementos Discretos de Refluxo de Material de Sustentação de Fraturas em Poços de Petróleo

Dissertação apresentada como requisito parcial para obtenção do título de Mestre pelo Programa de PósGraduação em Engenharia Civil da PUC-Rio. Área de concentração: Geotecnia

Orientador: Celso Romanel Co-orientadora: Deane Mesquita Roehl 


\section{Simulação 3D pelo Método dos Elementos Discretos de Refluxo de Material de Sustentação de Fraturas em Poços de Petróleo}

Dissertação apresentada como requisito parcial para obtenção do título de Mestre pelo Programa de PósGraduação em Engenharia Civil da PUC-Rio. Aprovada pela Comissão Examinadora abaixo assinada.

Prof. Celso Romanel

Orientador, PUC-Rio

Prof $^{a}$ Deane Mesquita Roehl Co-orientadora, PUC-Rio

Prof. Paulo Dore Fernandes CENPES - PETROBRAS

Prof $^{a}$ Christianne de Lyra Nogueira Universidade Federal de Ouro Preto

Prof. José Eugenio Leal Coordenador Setorial do Centro

Técnico Científico - PUC-Rio

Rio de Janeiro, 06 de outubro de 2008 
Todos os direitos reservados. É proibida a reprodução total ou parcial do trabalho sem autorização da universidade, do autor e do orientador.

\section{Carmen Julia Ayquipa Huamán}

Graduou-se em Engenharia Civil pela Universidad Nacional de Ingenieria (UNI) de Lima, Peru, em 1990. Ingressou em 2006 no curso de Mestrado em Engenharia Civil da Pontifícia Universidade Católica de Rio de Janeiro, na área de Geotecnia, onde desenvolveu dissertação de mestrado na linha de pesquisa geomecânica computacional.

Ficha Catalográfica

\section{Ayquipa Huaman, Carmen Julia}

Simulação 3D pelo Método dos Elementos Discretos de Refluxo de Material de Sustentação de Fraturas em Poços de Petróleo / Carmen Julia Ayquipa Huaman; orientador: Celso Romanel; co-orientadora: Deane Mesquita Roehl - Rio de Janeiro: PUC, Departamento de Engenharia Civil, 2008.

v., 1 131f.: il. ; $29.7 \mathrm{~cm}$

Dissertação (mestrado) - Pontifícia Universidade Católica do Rio de Janeiro, Departamento de Engenharia Civil.

Inclui referências bibliográficas.

1. Engenharia Civil - Teses. 2. Método dos elementos discretos. 3. Poços de petróleo. 4. Refluxo de propante 5. Fraturamento hidráulico. 6. Mecânica da partícula I. Romanel, Celso. II. Mesquita Roehl, Deane. III. Pontifícia Universidade Católica do Rio de Janeiro. Departamento de Engenharia Civil. IV. Título.Civil. III. Título. 


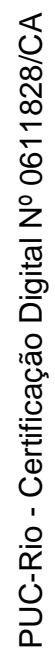

A meu amado esposo e filhos Aos meus queridos pais e irmãos 


\section{Agradecimentos}

A Deus de quem sou e a quem sirvo. Ele nos trouxe, nos sustentou e nos levará com bem.

Ao professor Celso Romanel pela oportunidade em desenvolver a presente pesquisa, pela orientação, amizade e apoio incondicional.

À Deane Mesquita Roehl pelo apoio.

A meu esposo David por todo o seu amor e apoio. A meus filhos Cinthia, Israel e Esteban, por terem me sustentado em oração, dia após dia, e pela alegria de viver.

À Itasca Consulting Group por terem me livremente proporcionado o software PFC3D que permitiu a elaboração desta dissertação. Obrigado a Loren Lorig, Peter Cundall, David Potyondy e Jason Furtney.

Aos pastores Luis Roberto Oliveira da Silva e Wilson Franklim e a toda a Igreja Batista de Vila Jaguaribe, pelo suporte moral, espiritual e material. Para nós, anjos de Deus.

À Jackeline, Kátia, Gladys, Gricel e Fanny pela amizade, e por terem me apoiado com a hospedagem e convivência durante as noites em que fiquei na Gávea. Ao Fabricio e Carlitos, amigos e companheiros da sala 608, bem como a todos os colegas da turma 2006.

À Luciana, Mariana, Paola e Wagner pelo auxílio na revisão deste trabalho.

À Rita e à Fátima pela grande ajuda e exemplos de eficiência no trabalho.

Ao CNPq e Capes pelo apoio financeiro durante o curso de pós-graduação.

Aos professores Celso Romanel e Deane Mesquita Roehl pelo auxílio financeiro que permitiu minha dedicação integral no desenvolvimento desta dissertação. 


\section{Resumo}

Huamán, Carmen Julia Ayquipa; Romanel, Celso (Orientador); Roehl, Deane Mesquita (Co-orientadora), Simulação 3D pelo Método dos Elementos Discretos de Refluxo de Material de Sustentação de Fraturas em Poços de Petróleo. Rio de Janeiro, 2008, 131p, Dissertação de Mestrado - Departamento de Engenharia Civil, Pontifícia Universidade Católica do Rio de Janeiro.

A técnica para estimulação de um campo de petróleo através de fraturamento hidráulico inclui a injeção, com o fluido de fraturamento, de um material para sustentação da abertura da fratura, denominado propante. Uma das dificuldades deste processo é o refluxo do material de sustentação para o interior do poço durante a fase de produção de óleo, ocasionando diversos problemas que podem chegar, em situações extremas, à interrupção definitiva da produção. $\mathrm{O}$ controle do fluxo de propante representa, portanto, um grande desafio para a indústria do petróleo. Alguns modelos empíricos - correlação Stimlab, modelo de cunha livre, modelo da velocidade mínima de fluidificação, modelo semimecânico - foram desenvolvidos para a previsão desse fenômeno, mas não contemplam todas as variáveis que influenciam no refluxo do material de sustentação da fratura. Uma alternativa aos modelos empíricos é utilizar o método dos elementos discretos para simular computacionalmente a instabilidade do pacote granular. Nesta dissertação busca-se modelar o comportamento do refluxo do propante utilizando uma modelagem 3D da fratura, partículas e fluxo de óleo com auxílio do programa computacional PFC3D baseado no método dos elementos discretos. Os dados das análises (tipos de propante, temperatura, características do fluido e do propante, etc) se referem a 4 cenários principais considerando fraturas sob diversos níveis de tensão e forças de arraste. Foram feitas discussões considerando os resultados numéricos obtidos por análises computacionais bi e tridimensionais, bem como comparações com modelos empíricos de previsão do refluxo do material de sustentação.

\section{Palavras - chave}

Método dos elementos discretos, poço de petróleo, refluxo de propante, fraturamento hidráulico, mecânica da partícula. 


\section{Abstract}

Huamán, Carmen Julia Ayquipa; Romanel, Celso (Advisor); Roehl, Deane Mesquita (Co-Advisor), 3D Simulation by the Discrete Element Method of Proppant Flowback along Fractures in Oil Wells, Rio de Janeiro, 2008. 131 p. MSc. Dissertation - Departamento de Engenharia Civil, Pontifícia Universidade Católica do Rio de Janeiro.

The technique for stimulating an oil field through hydraulic fracturing consists of pumping into the oil bore the fracturing fluid mixed with a material to hold the fracture open, called proppant. One of the difficulties in this process is the occurrence of proppant flowback into the well bore during oil production, causing several problems that can result, in critical situations, in a definitive interruption of the oil production. Control of proppant flowback represents a great challenge for the petroleum industry. Some empirical models - Stimlab correlation, proppedfree wedge model, minimum fluidization velocity, semi-mechanistic model - were developed for prediction of proppant flowback, yet they do not encompass all variable that play a role in this complex phenomenon. An alternative is to employ the discrete element method in order to computationally simulate the instability of the granular package. The main goal of this thesis is to investigate the proppant flowback phenomenon, through a 3D model of the fracture, particles and flow conditions using the computational program PFC3D, a code based on the discrete element method. The input data (type of proppant, temperature, characteristics of the fluid and proppant, etc.) relate to 4 main scenarios that consider fractures under several levels of stress closure and drag forces. The numerical results computed from 2D and 3D analyses were discussed, as well as comparisons were done with the predictions obtained with empirical methods.

\section{Keywords}

Discrete element method, oil bore, proppant flowback, hydraulic fracturing, particle mechanics. 


\section{Sumário}

1.0. INTRODUÇÃO 23

2.0. REFLUXO DO MATERIAL DE SUSTENTAÇÃO:

FATORES DETERMINANTES E MODELOS DE PREVISÃO 26

2.1. PRINCIPAIS FATORES QUE INFLUENCIAM NO REFLUXO 26

2.1.1. Largura da Fratura 26

2.1.2. Tensão de Fechamento 28

2.1.3. Forças de Arraste 35

2.2. MODELOS EMPÍRICOS PARA PREVISÃO DO REFLUXO 38

2.2.1. Correlação do Consórcio Stimlab 38

2.2.2. Modelo da Cunha Livre $\quad 39$

2.2.3. Modelo da Velocidade Mínima de Fluidificação 42

2.2.4. Modelo Semi-mecânico 43

2.2.5. Comparações entre os Modelos Empíricos 47

3.0 MÉTODO DOS ELEMENTOS DISCRETOS

E O PROGRAMA PFC

3.1. INTRODUÇÃO

3.2. MÉTODO DOS ELEMENTOS DISCRETOS 57

3.2.1. Equação do Movimento 58

3.2.2. Forças de Contato 59

3.2.3. Amortecimento Global 62

3.2.4. Integração Numérica 63

3.2.5. Algoritmo de busca por contatos 64

3.2.6. Relaçoes Força-Deslocamento 66

3.2.6.1. Modelo de Hooke 67

3.2.6.2. Modelo Simplificado de Hertz - Mindlin 67 
3.2.6.3. Modelo de Hooke - Coulomb

3.2.6.4. Modelo Frágil 70

3.2.6.5. Modelo Não Linear - Elástico 70

3.2.7. Acoplamento sólido - fluido 71

3.2.7.1 Abordagem em macro-escala 72

3.2.7.2. Abordagem em meso-escala 72

3.2.7.3. Abordagem em micro-escala 73

3.3. PROGRAMA PFC 73

3.3.1. Introdução 73

3.3.2. PFC2D e PFC3D 75

3.3.3. Características principais do PFC3D 76

3.3.4. Modelagem do fluido 80

3.3.4.1. Equação de interação fluido partícula 81

4.0. APLICAÇÃO DO PFC PARA ESTUDO DO

FENÔMENO DO REFLUXO DO PROPANTE 87

4.1. INTRODUÇÃO

4.2. CENÁRIO 1

4.2.1. Características 88

4.2.2. Modelagem utilizando os programas PFC2D e PFC3D 90

4.2.2.1. Geração de partículas 90

4.2.2.2. Condições de contorno 92

4.2.2.3. Tensão de Fechamento 93

4.2.2.4. Aplicação do Fluido 94

4.2.3. Comentario sobre a definição do modelo 3D 94

4.2.4. Comparações entre as modelagens 2D e 3D 96

4.2.5. Resultados 98

4.3. CENÁRIO 2

4.3.1. Características 101

4.3.2. Resultados 102

4.4. CENÁRIO 3

4.4.1. Características 105

4.4.2. Resultados 107

4.5. CENÁRIO 4 
4.5.1. Características 110

4.5.2. Resultados 110

4.6. ANÁLISE DOS RESULTADOS DE ESTABILIDADE DE TODOS OS CENÁRIOS 112

4.6.1. Forças de contato máximas 112

4.6.2. Gradientes de pressão máximos 114

4.6.3. Comparação com resultados da literatura 117

5. CONCLUSÕES E SUGESTÕES 119

5.1. CONCLUSÕES 119

5.2. SUGESTÕES 123

REFERÊNCIAS BIBLIOGRÁFICAS 124 


\section{Lista de Figuras}

Figura 1.1 - Esquema da técnica de fraturamento hidráulico 24 (EPA, 2004).

Figura 2.1 - Efeitos da tensão de fechamento na estabilidade 30 da fratura hidráulica (Canon, 2003).

Figura 2.1a - Condição 3aㅡ da figura 2.1 mostrando a ação de 30 forças mecânicas na direção do poço, originada por elevada tensão de fechamento, podem contribuir para o refluxo do material de sustentação da fratura.

Figura 2.2 - Ilustração da penetração do propante na rocha da 31 fomação (Barree et al., 2003).

Figura 2.3 - Comparação do embutimento do propantes 31 Interprop Plus 20/40, Jordan 20/40, e AcFrac CR 20/40 dentro do arenito Ohio, após $100 \mathrm{~h}$ usando o fluido de $2 \%$ de $\mathrm{KCl}$, para as tensões de fechamento e temperaturas indicadas (Penny, 1987).

Figura 2.4 - Esmagamento e penetração do propante na rocha 32 da formação (apud Legarth et al., 2003)

Figura 2.5 - Condutividade da fratura a longo prazo em função 33 da concentração de propante (apud WWW.carboceramics.com).

Figura 2.6 - Determinação experimental de valores de 33 concentração para assegurar estabilidade do pacote granular, considerando tensão de techamento da fratura no intervalo 2000 - 4500 psi (13,67 MPa - 30,75 MPa) na temperatura 120 ${ }^{\circ} \mathrm{C}$ (Haidar, 2003).

Figura 2.7 - Representação esquemática de pinch-out ( 34 Andrews e Hjorholt, 1998).

Figura 2.8 - Configuração do pacote granular após 12036 minutos de fluxo (vazão de $0.5 \mathrm{gpm}$ ) em fratura de 0.16 polegadas de largura, sob tensão de fechamento de 500 psi (Goel et al., 1999). 
Figura 2.9 - Célula de condutividade com retentor 37 transparente e $1 / 2$ polegada de perfuração utilizada para ensaios experimentais de fluxo de propante sob variações da tensão de fechamento, (Jim et al., 1999).

Figura 2.10 - Modelo de célula de condutividade 37 (transparente) com uma largura simulada de fratura de 3/8" (Batenburg et al., 1999).

Figura 2.11 - Modelo de célula de condutividade dividida em $2 \quad 37$ seções verticais (Batenburg et al., 1999).

Figura 2.12 - Representação gráfica do modelo Stimlab 39 delimitando regiões de estabilidade para diferentes números de camadas de grãos de propante no interior da fratura (Stimlab, 1996-2002).

Figura 2.13- Representação gráfica do modelo da cunha livre 41 (Andrews e Kjorholt,1998).

Figura 2.14 - Resultados experimentais de ensaios de 45 laboratório (Canon, 2003).

Figura 2.15 - Envoltórias para areia na malha 20/40, de acordo 48 ao modelo semi-mecánico, Canon (2003).

Figura 2.16 - Envoltórias para propante cerâmico de alta 48 resistência na malha 20/40, de acordo ao modelo Semimecânico, Canon (2003).

Figura 2.17 - Curvas do Modelo da Cunha Livre (apud Canon, 49 2003).

Figura 2.18 - Curvas do Modelo Semi-Mecânico (apud Canon, 49 2003).

Figura 3.1 - Resultados gráficos de simulação do DEM para 53 três larguras de fratura (Canon, 2003).

Figura 3.2 - Variação da força de contato normalizada média 54 com a largura normalizada da fratura.

Figura 3.3 - Variação da força de contato normalizada máxima 54 com a largura normalizada da fratura.

Figura 3.4 - Comportamento de material de sustentação para 56 
o caso 3c. A esquerda velocidades dos grãos; à direita, forças compressivas de contato, com a tonalidade clara representando grãos sem carregamento, (Asgian et al,,1995).

Figura 3.5 - Etapas Básicas de ciclo de cálculo do Método dos 57 Elementos Discretos.

Figura 3.6 - Representação do sistema de coordenadas global 58 e local.

Figura 3.7 - Variaveis que definem um contato. $\quad 60$

Figura 3.8 - Deformação de dois grãos em contato de acordo 68 com a teoria clássica de Hertz-Mindlin, (Shweiger, 2006).

Figura 3.9 - Rigidez normal Kn vs força normal fn no contato 69 entre grãos de areia média, (Shweiger, 2006).

Figura 3.10 - Modelo de Hooke - Coulomb para as direções 70 normal e tangencial.

Figura 3.11 - Modelos de rigidez e de deslizamento no contato 79 (manual de PFC3D).

Figura 3.12 - Modelos de Ligação (manual de PFC3D). 80

Figura 3.13 - Fluxo através do pacote de partículas (manual 81 PFC3D).

Figura 3.14 - Força de arraste em função do diâmetro do grão 83 e gradiente de pressão. (peneiras n. $16=0,047$ in., n. $20=$ 0,033 in. e n. $40=0,016$ in) - Asgian et al (1995).

Figura 3.15 - Fluxograma do processo de cálculo no programa 85 PFC3D com o esquema de fluido (manual do PFC3D).

Figura 3.16 - Fluxograma do esquema de fluido (manual do 86 PFC3D).

Figura 4.1 - Granulometria adotada para os cenários 1,2 e $4 . \quad 91$ Figura 4.2 - Geração de partículas (discos) pelo programa 91 PFC2D.

Figura 4.3 - Geração de partículas (esferas) pelo programa 92 PFC3D.

Figura 4.4 - Condições de contorno no problema 92 bidimensional. 
Figura 4.5 - Condições de contorno no problema 93 tridimensional.

Figura 4.6 - Fluxo de partículas pela aplicação da tensão de 93 fechamento (caso 2D).

Figura 4.7 - Fluxo de partículas pela aplicação da tensão de 94 fechamento (caso 3D).

Figura 4.8 - Vetores de velocidade do fluido na simulação 2D. 94

Figura 4.9 - Vetores de velocidade do fluido na simulação 3D. 94

Figura 4.10 - Resultados em $\mathrm{t}=2 \mathrm{~s}$ para o modelo com $\mathrm{h} / \mathrm{d}_{\mathrm{p}}=95$ 5

Figura 4.11 - Resultados em t=2 s para o modelo com $\mathrm{h} / \mathrm{d}_{\mathrm{p}}=95$ 10

Figura 4.12 - Resultados em $\mathrm{t}=2 \mathrm{~s}$ para o modelo com $\mathrm{h} / \mathrm{d}_{\mathrm{p}}=95$ 20 (vista lateral na figura superior e vista de topo na figura inferior).

Figura 4.13 - Velocidades do fluido ao longo do eixo da fratura 96 em três pontos do pacote granular, determinados com os programas PFC2D e PFC3D.

Figura 4.14 - Forças de arraste aplicadas nas partículas em 100 função do gradiente de pressão ao longo do eixo da fratura, nas regiões posterior, central e próxima ao poço, nas variações do cenário 1.

Figura 4.15 - Forças de arraste aplicadas nas partículas em 104 função do gradiente de pressão ao longo do eixo da fratura, nas regiões posterior, central e próxima ao poço, nas variações do cenário 2.

Figura 4.16 - Granulometria do material de sustentação no 106 cenário 3.

Figura 4.17 - Forças de arraste aplicadas nas partículas em 109 função do gradiente de pressão ao longo do eixo da fratura, nas regiões posterior, central e próxima ao poço, nas variações do cenário 3 .

Figura 4.18 - Forças de arraste aplicadas nas partículas em 112 função do gradiente de pressão ao longo do eixo da fratura, 
nas regiões posterior, central e próxima ao poço, nas variações do cenário 4 .

Figura 4.19 - Máximas forças de contato para todas as 113 análises dos cenários 1, 2, 3 e 4.

Figura 4.20 - Variações das larguras normalizadas de 113 instabilidade com tensão de fechamento (sem fluxo).

Figura 4.21 - Curvas de instabilidade do pacote granular, em 115 função dos gradientes de pressão, tensão de fechamento e largura normalizada da fratura. Os símbolos vazados indicam ocorrência de instabilidade.

Figura 4.22 - Comparação da forças de arraste na região 116 posterior do modelo da fratura.

Figura 4.23 - Granulometrias usadas na presente dissertação 118 (cenários 1,2,3 e 4) e no trabalho de Asgian et al (1995). 


\section{Lista de tabelas}

Tabela 2.1 - Equivalência entre a largura normalizada da fratura e a concentração do propante por área tratada da 27 fratura (Milton-Tayler et al., 1992).

Tabela 2.2 - Valores típicos de resistencia nominal do propante Smax (Canon, 2003).

Tabela 2.3 - Estimativas de erro entre o modelo semimecânico e a correlação Stimlab (Canon, 2003).

Tabela 3.1 - Características das simulações numéricas de Asgian et al (1995)

Tabela 3.2 - Propriedades geotécnicas de uma areia média.

Tabela 3.3 - Número máximo de partículas no PFC3D em função da memoria RAM disponível.

Tabela 4.1 - Características do Material de Sustentação dos Cenários 1,2 e 4.

Tabela 4.2 - Características do Material de Sustentação do Cenários 3.

Tabela 4.3 - Características da Formação do cenário 1

Tabela 4.4 - Características do Poço Vertical do cenário 1

Tabela 4.5 - Características do Fluido do cenário 1

Tabela 4.6 - Características da Fratura do cenário 1

Tabela 4.7 - Granulometria utilizada nos cenários 1,2 e 4

Tabela 4.8 - Comparação entre os modelos 2D e 3D para o cenário 1.

Tabela 4.9 - Resultados da análise de estabilidade por modelos empíricos para o cenário 1, Velozo (2006).

Tabela 4.10 - Análises de estabilidade do pacote granular com variações do cenário 1 
Tabela 4.11 - Características da Formação do cenário $2 \quad 101$

Tabela 4.12 - Características do Poço Vertical do cenário 2101

Tabela 4.13 - Características do Fluido do cenário 2

Tabela 4.14 - Características da Fratura do cenário $2 \quad 102$

Tabela 4.15 - Resultados da análise de estabilidade por modelos empíricos para o cenário 2, Velozo (2006).

Tabela 4.16 - Análises de estabilidade do pacote granular com variações do cenário 2

Tabela 4.17 - Granulometria do material de sustentação no cenário 3

Tabela 4.18 - Características da Formação do cenário 3

Tabela 4.19 - Características do Poço Vertical do cenário 3106

Tabela 4.20 - Características do Fluido do cenário $3 \quad 106$

Tabela 4.21 - Características da Fratura do cenário $3 \quad 107$

Tabela 4.22 - Resultados da análise de estabilidade por modelos empíricos para o cenário 3, Cachay (2005).

Tabela 4.23 - Análises de estabilidade do pacote granular com variações do cenário 3

Tabela 4.24 - Características da Formação do cenário 4

Tabela 4.25 - Características do Poço Vertical do cenário 4110

Tabela 4.26 - Características do Fluido do cenário $4 \quad 110$

Tabela 4.27 - Análises realizados no Cenário 4

Tabela 4.28 - Comparação entre modelos para previsão do refluxo de material de sustentação 


\section{LISTA DE SÍMBOLOS}

Alfabeto Latino

$a^{o} \quad$ : Raio de superficie de contato circular plana;

$a^{\prime} \quad$ : Constante para o modelo Semi-Mecânico = 7,7172;

$A_{f} \quad$ : Termo para o cálculo da velocidade mínima do fluidização;

Bf : Termo para o cálculo da velocidade mínima do fluidização;

$C \quad$ : Termo do fechamento no modelo de PFW, $\mathrm{psia}^{-1}$;

$C^{i} \quad$ : Centro da partícula i;

$C^{j} \quad$ : Centro da partícula j;

$C_{f} \quad$ : Termo para o cálculo da velocidade mínima do fluidização;

$C_{D} \quad$ : Coeficiente de arraste para partículas esfêricas;

$c \quad$ : Coesão;

$\mathrm{C}_{\mathrm{d}} \quad$ : Condutividade;

Co : Fator de coesão, usado pelo consorcio Stimlab;

$C_{p} \quad$ : Concentração real do propante, $\mathrm{lb} / \mathrm{ft}_{2}$;

$D_{C^{i} C^{j}} \quad$ : Distância entre centros de dos círculos i e j:

d : Diâmetro da partícula;

: Distancia correspondente ao ponto no qual $50 \%$ da força máxima $\left(\mathrm{P}_{\mathrm{o}}\right)$ é atingida

$d_{p i} \quad$ : Diâmetro das $\mathrm{n}_{\mathrm{p}}$ partículas no interior do volume $\Delta V$;

$\bar{d}_{p} \quad$ : Diâmetro médio das partículas;

$D^{\prime}{ }_{p} \quad$ :Diâmetro da partícula em micrones, $\mu$

$D \quad$ : Diámetro esférico, modelo de Cunha livre;

$d_{p} \quad$ : Diâmetro médio do grão de propante, in;

$d P / d x \quad$ : Gradiente de pressão, psi/ft;

$d_{t} \quad$ : Distância entre dos partículas

$d_{\text {ref }} \quad$ : Diâmetro da referência, 0,0284 in;

E :Módulo de Young

$E_{p} \quad$ :Módulo de Young de partículas esféricas;

$F \quad$ : Termo de araste, $\mathrm{psi} / \mathrm{ft}$;

$f^{i} \quad$ : Vetor força, representa as forças (Fx,Fy, Fz) e momentos (Mx, My, Mz)

$f_{n} \quad$ : Força normal no contato; 
$f_{\text {ext }} \quad$ : Forças e momentos devido a cargas externas;

$f_{\text {int }}$ : Forças e momentos resultantes das interações com outras partículas ou obstáculos;

$f_{\text {dam }}$ : Parcela originária das forças de amortecimento do sistema ou amortecimento global;

$F_{\text {dsum }} \quad$ : Soma das forças de arraste;

$F_{d i j} \quad$ : Força de arraste aplicada à partícula i, na direção j;

$F_{d i x} \quad:$ Força de arraste aplicada à partícula i;

$f_{\text {intx }}$ : Força de interação por unidade de volume entre as partículas e o fluido na direção x;

$f_{\text {intj }} \quad$ : Força de interação por unidade de volume na direção j;

$F_{e x} \quad$ : Gradiente de pressão na qual ocorre o movimento dos grãos em laboratório;

$F_{M A X} \quad$ : O máximo gradiente estável de pressão para desestabilizar grãos de propante fluxos, psi/ft;

$F_{m} \quad$ : Gradiente de pressão crítico previsto por um modelo;

Ft : Força tangencial;

: Mínimo gradiente de pressão suficiente para desestabilizar os grãos de

$F_{F V}$ propante (psi/ft);

$F_{\text {sta }}$ : Gradiente da pressão estável máximo no modelo Semi-Mecânico, psi/ft;

G : Módulo de cisalhamento;

$h_{f} \quad:$ Altura da fratura, $\mathrm{ft}$;

IP : Índice de produtividade;

$K \quad$ : Permeabilidade da formação;

kf : Permeabilidade do pacote granular;

$k_{n} \quad$ : Rigidez de contato na direção perpendicular à superfície;

$k^{\text {rot }} \quad$ : Rigidez rotacional;

$k_{s} \quad:$ Rigidez de contato na direção tangencial de contato;

$k_{\text {tran }} \quad:$ Rigidez traslacional;

$L \quad$ : Comprimento da fratura;

$\mathrm{L} / \mathrm{d}_{\mathrm{p}} \quad$ : Comprimento de largura normalizado;

$m$ : Massa do grão;

$\mathrm{m}^{\mathrm{i}} \quad$ : massa da partícula i;

n : Porosidade;

$\mathrm{n}_{\mathrm{p}} \quad$ : Número de partículas no interior do volume;

$\bar{n} \quad$ : Vetor normal;

$P_{e} \quad$ : Pressão do reservatório (pressão estática); 
$P_{c, \text { net }} \quad$ : Tensão de fechamento efetiva;

$P_{o} \quad$ : Valor máximo de dt;

$\Delta V \quad$ : Volume de controle fixo;

$\nabla P \quad:$ Gradiente de pressão ;

$\nabla P_{j} \quad$ : Gradiente de pressão na direção j;

$p_{p} \quad$ : Poro pressão;

$P_{w f} \quad$ : Pressão do fluido no fundo do poço;

$Q_{p} \quad$ : Vazão de produção;

$r c^{i} \quad$ : Vetor que une o centro da partícula $\mathrm{C}^{\mathrm{i}}$ ao ponto de contato $\mathrm{C}$;

$r c^{j} \quad:$ Vetor que une o centro da partícula $\mathrm{C}^{\mathrm{j}}$ ao ponto de contato $\mathrm{C}$;

$r \quad$ : Raio da partícula;

$r_{e} \quad$ : Raio do reservatório;

Re : Número de Reynolds;

$R^{i} \quad$ : Raio da partícula i;

$R^{j} \quad$ : Raio da partícula j;

$S G_{p} \quad$ : Densidade do grão;

$S_{M A X} \quad$ : Resistência nominal do propante em psia;

$S_{t} \quad$ : Termo da resistência, modelo Semi-mecânico;

$T$ : Temperatura;

$\Delta t \quad$ : Passo de tempo;

$t \quad:$ tempo t qualquer;

$t_{\text {crit }} \quad:$ tempo crítico;

$u_{j} \quad$ : Velocidade do fluido na direção j

$\ddot{u}_{i} \quad$ : Aceleração da partícula i;

$u_{r n} \quad$ : Deslocamento relativo normal;

$u_{r n \theta} \quad$ : Parcela normal de deslocamento;

$u_{r t} \quad$ : Deslocamento relativo tangencial;

$u_{\theta}^{i} \quad$ : Deslocamento traslacional;

$u_{\theta t}^{i} \quad$ : Parcela de deslocamento tangencial devida à rotação $\theta^{i}$ da partícula i;

$V_{c} \quad$ : Velocidade crítica do consorcio Stimlab, $\mathrm{ft} / \mathrm{s}$;

$V_{c, s} \quad$ : Velocidade crítica normalizada da companhia Stimlab, ft/s; 
$v \quad$ : Velocidade aparente do fluido:

$v_{f} \quad:$ Velocidade mínima do fluidificação, $\mathrm{ft} / \mathrm{s}$;

$\mathrm{v}_{\mathrm{rn}} \quad$ : Velocidade relativa normal;

$v^{i} \quad$ : Velocidade traslacional da partícula i;

$\nu^{j} \quad:$ Velocidade traslacional da partícula $\mathrm{j} ;$

$v_{r} \quad$ : Velocidade relativa;

$v_{\theta}^{i} \quad$ : Velocidade traslacional da partícula i;

$v_{r n \theta} \quad:$ Parcela normal de velocidade;

: Parcela de velocidade tangencial devida à velocidade rotacional $\omega^{\mathrm{i}} \mathrm{da}$ partícula i;

$v_{r t} \quad$ : Velocidade relativa tangencial;

$\bar{v}_{j} \quad$ : Velocidade média das partículas na direção j;

W : Largura da fratura;

$W_{r} \quad$ : Largura normalizada;

$W_{T} \quad$ : Termo da largura no modelo Semi-Mecanico, in;

$w^{o} \quad$ : Deformação do grão;

\section{Alfabeto grego}

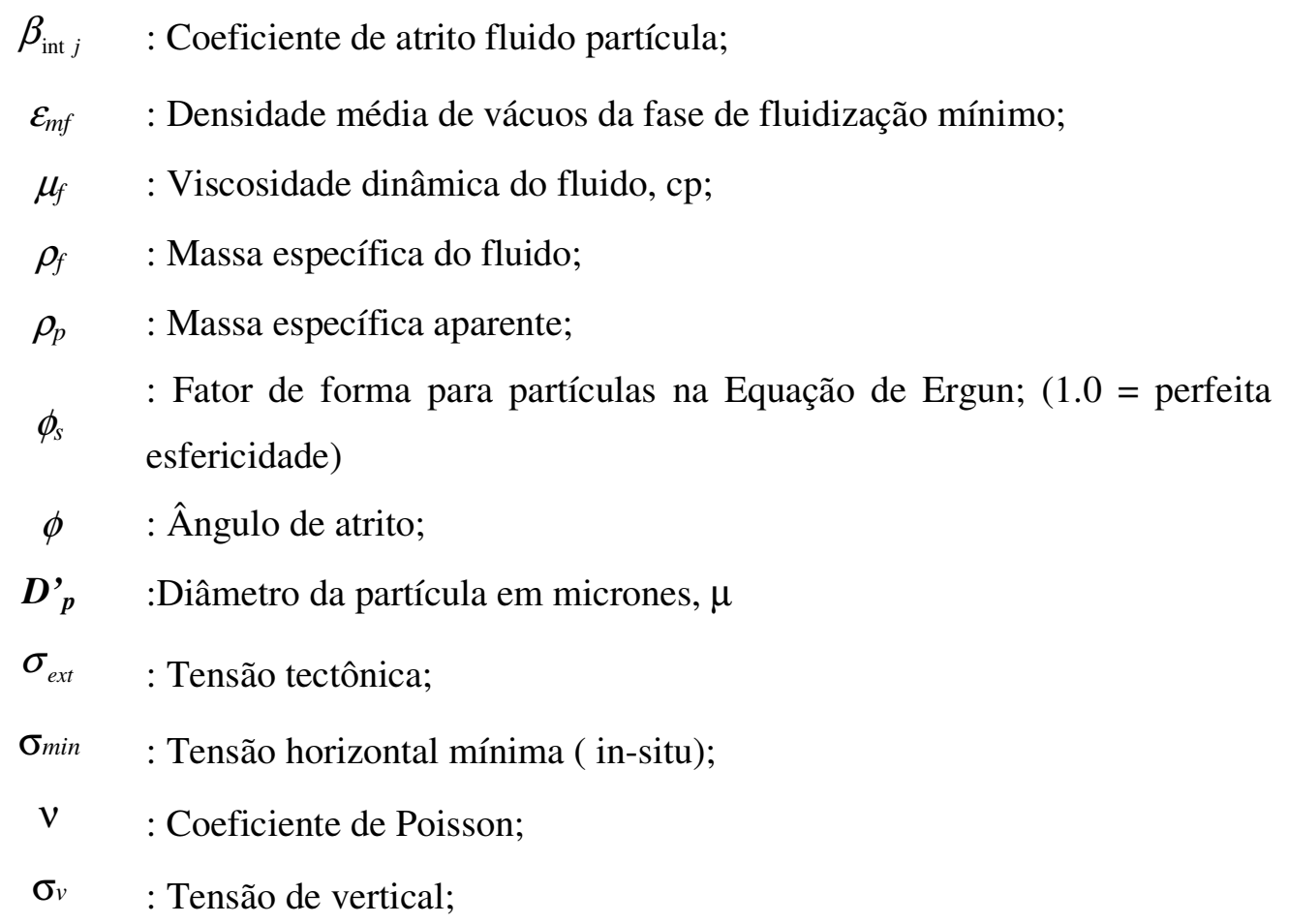


$\alpha \quad$ : Constante de Biot.

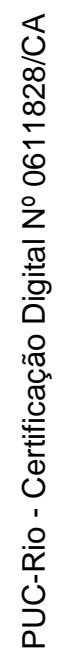

